\title{
Abstracts
}

Human birth observed in real-time open magnetic resonance imaging

Presented at Birth: Clinical Challenges in Labor and Delivery, presented by the Division of Continuing Medical Education, Wayne State University School of Medicine, Chicago, IL, Sept. 9-11, 2011.

Christian Bamberg, MDaㅡ, Grit Rademachera, Felix Güttler $\underline{\underline{b}}$, Ulf Teichgräber, PhD $\underline{\underline{b}}$, Malte Cremer, MD드, Christoph Bührer, PhD드, Claudia Spies, PhD $\underline{d}$, Larry Hinkson, MDạ, Wolfgang Henrich, PhDạa, Karim D. Kalache, PhD $\underline{a}$, Joachim W. Dudenhausen, PhD $\underline{a}$

Objective: Knowledge about the mechanism of labor is based on assumptions and radiographic studies performed decades ago. The goal of this study was to describe the relationship between the fetus and the pelvis as the fetus travels through the birth canal, using an open magnetic resonance imaging (MRI) scanner.

Study Design: The design of the study used a realtime MRI series during delivery of the fetal head.

Results: Delivery occurred by progressive head extension. However, extension was a very late movement that was observed when the occiput was in close contact with the inferior margin of the symphysis pubis, occurring simultaneously with gliding downward of the fetal head.

Conclusion: This observational study shows, for the first time, that birth can be analyzed with real-time MRI. MRI technology allows assessment of maternal and fetal anatomy during labor and delivery.

Early menopause and risk of osteoporosis, fracture and mortality: a 34-year prospective observational study in $\mathbf{3 9 0}$ women

O Svejme, HG Ahlborg, J-Å Nilsson, MK Karlsson

Objective: A prospective evaluation of the long-term effects of early menopause on mortality, risk of fragility fracture and osteoporosis.

Design: Prospective population-based observational study.

Setting: Malmö, Sweden.

Population: A total of 390 white north European women aged 48 years at the start of the study.
Methods: At baseline, bone mineral density (BMD) was measured by single-photon absorptiometry (SPA) in the distal forearm and menopausal status was noted. Menopause was determined according to the World Health Organization criterion of a minimum of 12 months of continuous amenorrhoea. Women were divided into early menopause (occurring before age 47 years) and late menopause (occurring at age 47 years or later). At age 77, forearm BMD was remeasured by SPA and proximal femur and lumbar spine BMD were measured by dual-energy $X$-ray absorptiometry (DXA). The prevalence of osteoporosis was determined using the DXA data. Mortality rate and the incidence of fractures were registered up until age 82. Data are presented as means with 95\% confidence intervals $(95 \% \mathrm{Cl})$.

Main outcome measures: Incidence of fragility fractures, mortality, prevalence of osteoporosis at age 77.

Results: Women with early menopause had a risk ratio of $1.83(95 \% \mathrm{Cl} 1.22-2.74)$ for osteoporosis at age 77 , a risk ratio of $1.68(95 \% \mathrm{Cl} 1.05-2.57)$ for fragility fracture and a mortality risk of $1.59(95 \% \mathrm{Cl}$ 1.04-2.36).

Conclusions: Menopause before age 47 is associated with increased mortality risk and increased risk of sustaining fragility fractures and of osteoporosis at age 77 .

Acceptability of misoprostol-only medical termination of pregnancy compared with vacuum aspiration: an international, multicentre trial

MI Rodriguez, A Seuc, N Kapp, H von Hertzen, NTM Huong, D Wojdyla, S Mittal, K Arustamyan, R Shah, the World Health Organization Research Group on Post-Ovulatory Methods of Fertility Regulation ${ }^{\dagger}$

Objective: To compare women's acceptance of misoprostol-only medical termination of pregnancy (TOP) with surgical TOP.

Design: Prospective cohort study.

Setting :Termination of pregnancy clinics in New Delhi, Mumbai, Hanoi, Tbilisi, Trivandrum and Yerevan.

Population: Women requesting TOP, at 63 days of gestation or less, at study sites where both medical and surgical methods were available. 
Methods Serial surveys eliciting measures of women's satisfaction and acceptance of TOP method were administered. Data were analysed using crosstabulation and logistic regression to determine if TOP method was predictive of acceptability.

Main outcome measures Patient acceptance.

Results High acceptability of both surgical and misoprostol-only TOP.

Conclusions Where medical TOP with mifepristone is not available, misoprostol-only medical TOP is acceptable to women who have the choice between medical or surgical techniques.

Prediction of spontaneous preterm delivery in women with threatened preterm labour: a prospective cohort study of multiple proteins in maternal serum

P Tsiartas ${ }^{1,2}$, RM Holst ${ }^{2}$, UB Wennerholm², H Hagberg $^{2,3}$, DM Hougaard ${ }^{4}$, K Skogstrand ${ }^{4}$, BD Pearce $^{5}$, P Thorsen 6 , M Kacerovsky²,7, B Jacobsson $^{2,8}$

Objective To analyse whether specific proteins in maternal serum and cervical length, alone or in combination, can predict the likelihood that women with intact membranes with threatened preterm labour will deliver spontaneously within 7 days of sampling.

Design Cohort study.

Setting Sahlgrenska University Hospital, Gothenburg, Sweden.

Population Women at between 22 and 33 weeks of gestation with threatened preterm labour $(n=142)$ admitted to the Sahlgrenska University Hospital, Gothenburg, Sweden, in 1995-2005.

Methods Maternal serum was tested for 27 proteins using multiplex xMAP technology. Individual levels of each protein were compared, and calculations were performed to investigate potential associations between different proteins, cervical length and spontaneous preterm delivery. Receiver operating characteristic curves were used to find the best cutoff values for continuous variables in relation to spontaneous preterm delivery within 7 days of sampling. Prediction models were created based on a stepwise logistic regression using binary variables.

Main outcome measure Spontaneous preterm delivery within 7 days.
Results In order to determine the best prediction model, we analysed models of serum proteins alone, cervical length alone, and the combination of serum proteins and cervical length. We found one multivariable combined model through the data analysis that more accurately predicted spontaneous preterm delivery within 7 days. This model was based on serum interleukin-10 (IL-10) levels, serum RANTES levels and cervical length (sensitivity $74 \%$, specificity $87 \%$, positive predictive value $76 \%$, negative predictive value $86 \%$, likelihood ratio 5.8 and area under the curve 0.88).

Conclusions A combination of maternal serum proteins and cervical length constituted the best prediction model, and would help determine whether women with threatened preterm labour are likely to deliver within 7 days of measurement.

Depot medroxyprogesterone acetate and epithelial ovarian cancer: a multicentre casecontrol study

S Wilailak ${ }^{1}$, C Vipupinyo ${ }^{2}$, V Suraseranivong ${ }^{3}, \mathrm{~K}$ Chotivanich $^{4}$, C Kietpeerakool ${ }^{5}, \mathrm{Y}_{\text {Tanapat }}{ }^{6}, \mathrm{~S}$ Therasakvichya $^{7}$, S Hamontri ${ }^{8}, V$ Linasmita $^{1}$, S Bunyapipat $^{9}, \mathrm{~S}$ Chindavijak $^{10}, \mathrm{~K}$ Ittiwisavakul ${ }^{11}, \mathrm{~N}$ Khemapech ${ }^{12}$, P Suekwattana ${ }^{13}$, D Thanapprapasr ${ }^{1}$, P Lumbiganon $^{1}$

Objective To evaluate the effect of depot medroxyprogesterone acetate (DMPA) in protecting against epithelial ovarian cancer (EOC) and to evaluate factors associated with the risk of EOC.

Design A multicentre, case-control study.

Setting Twelve hospitals located across Thailand.

Population Three hundred and thirty patients with EOC ('cases') and 982 matched controls were recruited from the 12 hospitals. Cases were newly diagnosed patients with EOC, demonstrated pathologically. Controls were age-matched patients admitted to different wards in the same hospital.

Methods Cases and controls were interviewed by trained interviewers using a standardised pre-tested questionnaire. The factors associated with EOC were evaluated using univariate and multivariate analyses.

Main outcome measures The odds ratio (OR) and 95\% confidence interval $(95 \% \mathrm{Cl})$ were calculated to assess the relationship between DMPA and EOC. 
Results The use of DMPA was found to be associated with a $39 \%$ reduction in the risk of EOC with an OR of 0.61 and a $95 \% \mathrm{Cl}$ of $0.44-0.85(P=0.002)$. A significant risk reduction (83\%) was observed when the duration of DMPA use was $>3$ years (OR 0.17 ; 95\% Cl 0.07-0.39; $P<0.001)$. Other factors associated with a reduced risk of EOC were the use of combined oral contraceptive pills and breastfeeding. A factor associated with an increased risk of EOC was a family history of gynaecological cancer.

Conclusions The results suggest that DMPA may have a protective effect against EOC. If this effect is real, then it represents an important non-contraceptive benefit of DMPA.

\section{Cost-effectiveness of cervical cancer screening: cytology versus human papillomavirus DNA testing}

J van Rosmalen, IMCM de Kok, M van Ballegooijen

Objective To determine the most cost-effective screening programme for cervical cancer.

Design Cost-effectiveness analysis from a societal perspective.

Setting The Netherlands.

Population Dutch women who have not been invited for human papillomavirus (HPV) vaccination.

Methods We calibrated the microsimulation screening analysis (MISCAN) model to Dutch epidemiological data. We used this model to consider nine screening strategies that use: (i) cytological testing with cytology triage for borderline/mildly abnormal smears; (ii) HPV testing with cytology triage for HPV-positive smears; or (iii) cytological testing with HPV triage for borderline/ mildly abnormal smears. For each strategy, we varied the number of screening rounds, the time interval, the age of the first screening, and the type of cytological testing (conventional or liquid-based cytology).

Main outcome measures Quality-adjusted life years (QALYS) gained and costs from a societal perspective.

Results Under the base-case assumptions, primary HPV testing with cytology triage is the most costeffective strategy. Using cost-effectiveness thresholds of $\cdot 20000$ and $\cdot 50000$ per QALY gained yields optimal screening programmes with three and seven screening rounds, respectively. The results are sensitive to several uncertain model inputs, most importantly the costs of the HPV test. For women aged 32 years or younger, primary cytology screening is more cost-effective than primary HPV testing.

Conclusions Increasing the interval between screening rounds and changing the primary test from cytology to HPV testing can improve the effectiveness and decrease the costs of cervical cancer screening in the Netherlands.

Pulmonary hypertension and pregnancy-a review of 12 pregnancies in nine women

RA Curry ${ }^{1}$, C Fletcher $^{2}$, E Gelson ${ }^{1}$, MA Gatzoulis ${ }^{2}$, M Woolnough ${ }^{3}$, N Richards ${ }^{3}$, L Swan ${ }^{2}$, PJ Steer ${ }^{1}$, MR Johnson ${ }^{1}$

Objective To report outcomes in a recent series of pregnancies in women with pulmonary hypertension $(\mathrm{PH})$.

\section{Design Retrospective case note review.}

Setting Tertiary referral unit (Chelsea and Westminster and Royal Brompton Hospitals).

Sample Twelve pregnancies in nine women with $\mathrm{PH}$ between 1995 and 2010.

Methods Multidisciplinary review of case records.

Main outcome measures Maternal and neonatal mortality and morbidity.

Results There were two maternal deaths (1995 and 1998), one related to pre-eclampsia and one to arrhythmia. Maternal morbidity included postpartum haemorrhage (five cases), and one post-caesarean evacuation of a wound haematoma. There were no perinatal deaths, nine live births and three firsttrimester miscarriages. Mean birthweight was $2197 \mathrm{~g}$, mean gestational age was 34 weeks (range 26-39), and mean birthweight centile was 36 (range 5-60). Five babies required admission to the neonatal intensive care unit, but were all eventually discharged home. All women were delivered by caesarean section (seven elective and two emergency deliveries), under general anaesthetic except for one emergency and one elective caesarean performed under regional block.

Conclusions Maternal and fetal outcomes for women with $\mathrm{PH}$ may be improving. However, the risk of maternal mortality remains significant, so that early and effective counselling about contraceptive options 
and pregnancy risks should continue to play a major role in the management of such women when they reach reproductive maturity.

\section{Glucose challenge test for detecting gestational diabetes mellitus: a systematic review}

M van Leeuwen ${ }^{1}$, MD Louwerse ${ }^{2}$, BC Opmeer $^{3}, \mathrm{~J}$ Limpens $^{4}$, MJ Serlie ${ }^{5}$, JB Reitsma ${ }^{3}$, BWJ Mol ${ }^{1}$

Background The best strategy to identify women with gestational diabetes mellitus (GDM) is unclear.

Objectives To perform a systematic review to calculate summary estimates of the sensitivity and specificity of the 50-g glucose challenge test for GDM.

Search strategy Systematic search of MEDLINE, EMBASE and Web of Science.

Selection criteria Articles that compared the 50-g glucose challenge test with the oral glucose tolerance test (OGTT, with a 75- or 100-g reference standard) before 32 weeks of gestation.

Data collection and analysis Summary estimates of sensitivity and specificity, with 95\% confidence intervals and summary receiver operating characteristic curves, were calculated using bivariate random-effects models. Two reviewers independently selected articles that compared the $50 \mathrm{~g}$ glucose challenge test to the oral glucose tolerance test (OGTT, 75 or 100 gram, reference standard) before 32 weeks of gestation.

Main results Twenty-six studies were included (13 564 women). Studies that included women with risk factors showed a pooled sensitivity of the $50-\mathrm{g}$ glucose challenge test of 0.74 (95\% $\mathrm{Cl} 0.62-0.87)$, a pooled specificity of $0.77(95 \% \mathrm{Cl} 0.66-0.89)$ (threshold value of $7.8 \mathrm{mmol} / \mathrm{l}$ ), a derived positive likelihood ratio (LR) of $3.2(95 \% \mathrm{Cl} 2.0-5.2)$ and a negative LR of 0.34 (95\% Cl 0.22-0.53). In studies with consecutive recruitment, the pooled sensitivity was $0.74(95 \% \mathrm{Cl} 0.62-0.87)$ for a specificity of 0.85 (95\% $\mathrm{Cl} 0.80-0.91$ ), with a derived positive LR of 4.9 $(95 \% \mathrm{Cl} 3.5-7.0)$ and negative LR of $0.31(95 \% \mathrm{Cl}$ $0.20-0.47$ ). Increasing the threshold for disease (OGTT result) increased the sensitivity of the challenge test, and decreased the specificity.

Author's conclusions The $50-\mathrm{g}$ glucose challenge test is acceptable to screen for GDM, but cannot replace the OGTT. Further possibilities of combining the 50-g glucose challenge test with other screening strategies should be explored.
Plasma uric acid remains a marker of poor outcome in hypertensive pregnancy: a retrospective cohort study

TL-A Hawkins ${ }^{1,2}$, JM Roberts ${ }^{3}$, GJ Mangos ${ }^{2,4}$, GK Davis $^{4}$, LM Roberts ${ }^{4}$, MA Brown ${ }^{2,4}$

Objective To examine the relationship between hyperuricaemia, haemoconcentration and maternal and fetal outcomes in hypertensive pregnancies.

Design Retrospective analysis of a database of hypertensive pregnancies.

Setting St George Hospital, a major obstetric unit in Australia.

Population A cohort of 1880 pregnant women without underlying hypertension or renal disease, referred for management of pre-eclampsia or gestational hypertension.

Methods Demographic, clinical and biochemical data at time of referral and delivery were collected for each pregnancy. Women were grouped according to diagnosis (pre-eclampsia or gestational hypertension) and logistic regression analysis was used to determine the relationship between uric acid, haemoglobin, haematocrit and adverse outcomes; an á level of $P<0.01$ was used for statistical significance.

Main outcome measures Composites of adverse maternal and fetal outcomes.

Results In women with 'benign' $\mathrm{GH}$ (without proteinuria or any other maternal clinical feature of pre-eclampsia) gestation-corrected hyperuricaemia was associated with increased risk of a small-for-gestational-age infant (OR 2.5; 95\% Cl 1.3-4.8) and prematurity (OR 3.2; $95 \% \mathrm{Cl} 1.4-7.2)$, but not with adverse maternal outcome. In the whole cohort of hypertensive pregnant women (those with pre-eclampsia or gestational hypertension) the risk of adverse maternal outcome (OR 2.0; 95\% Cl 1.6-2.4) and adverse fetal outcome (OR 1.8; 95\% Cl 1.5-2.1) increased with increasing concentration of uric acid. Hyperuricaemia corrected for gestation provided additional strength to these associations. Haemoglobin and haematocrit were not associated with adverse pregnancy outcome.

Conclusions Hyperuricaemia in hypertensive pregnancy remains an important finding because it identifies women at increased risk of adverse maternal and particularly fetal outcome; the latter, even in women with gestational hypertension without any other feature of pre-eclampsia. 


\section{Evolution of surgical management of early-stage endometrial cancer}

Presented at the 39th Global Congress of Minimally Invasive Gynecology, American Association of Gynecologic Laparoscopists, Las Vegas, NV, Nov. 812, 2010.

Objective We sought to examine the evolution of surgical care for early-stage endometrial cancers and factors affecting use of laparoscopy.

Study Design Women with surgically managed earlystage endometrial cancer were divided into 2 groups corresponding to before and after addition of faculty with formal fellowship training in laparoscopic staging and access to a robotic surgery platform.

Results In all, 502 women were identified. Laparoscopic management increased from 24-69\% between time periods $(P<.0001)$. Performance of comprehensive surgical staging, and lymph node counts, increased $(P<.0001)$ despite an increase in median body mass index $(P=.001)$. A traditional "straight stick" technique was performed in $72 \%$ of laparoscopic cases during the later period. Laparoscopy patients had lower estimated blood losses and shorter hospital stays (each $P<.0001$ ) compared to laparotomy patients.

Conclusion Addition of faculty with formal fellowship training in laparoscopic staging and access to a robotic surgery platform shifted management of early-stage endometrial cancer toward laparoscopy.

\section{Doppler assessment of the fetus with intrauterine growth restriction}

Society for Maternal-Fetal Medicine Publications Committee, Eliza Berkley, MD, Suneet P. Chauhan, MD, Alfred Abuhamad, MD,

Society for Maternal-Fetal Medicine Publications Committee, with the assistance of Eliza Berkley, Suneet P. Chauhan, and Alfred Abuhamad, Division of Maternal-Fetal Medicine at Eastern Virginia Medical School, Norfolk, VA

Received 11 January 2012. Accepted 12 January 2012. Available online 30 March 2012.

Objective We sought to provide evidence-based guidelines for utilization of Doppler studies for fetuses with intrauterine growth restriction (IUGR).
Methods Relevant documents were identified using PubMed (US National Library of Medicine, 1983 through 2011) publications, written in English, which describe the peripartum outcomes of IUGR according to Doppler assessment of umbilical arterial, middle cerebral artery, and ductus venosus. Additionally, the Cochrane Library, organizational guidelines, and studies identified through review of the above were utilized to identify relevant articles. Consistent with US Preventive Task Force suggestions, references were evaluated for quality based on the highest level of evidence, and recommendations were graded.

Results and Recommendations Summary of randomized and quasirandomized studies indicates that, among high-risk pregnancies with suspected IUGR, the use of umbilical arterial Doppler assessment significantly decreases the likelihood of labor induction, cesarean delivery, and perinatal deaths (1.2\% vs $1.7 \%$; relative risk, $0.71 ; 95 \%$ confidence interval, $0.52-0.98$ ). Antepartum surveillance with Doppler of the umbilical artery should be started when the fetus is viable and IUGR is suspected. Although Doppler studies of the ductus venous, middle cerebral artery, and other vessels have some prognostic value for IUGR fetuses, currently there is a lack of randomized trials showing benefit. Thus, Doppler studies of vessels other than the umbilical artery, as part of assessment of fetal well-being in pregnancies complicated by IUGR, should be reserved for research protocols.

\section{Autism risk in small- and large-for-gestational- age infants}

Presented at the 32nd annual meeting of the Society for Maternal-Fetal Medicine, Dallas, TX, Feb. 6-11, 2012.

Gaea Schwaebe Moore, MDㅁa $\underline{\mathrm{d}}$, Anna Weber Kneitel,

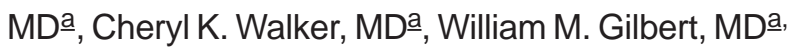

$\underline{\mathrm{b}}$, Guibo Xing, PhD $\underline{\mathrm{a}}, \underline{\mathrm{c}}$

Objective We sought to determine whether smallfor-gestational age (SGA) and large-for-gestational age (LGA) birthweights increase autism risk.

Study Design This was a retrospective cohort analysis comparing children with autism $(n=20,206)$ within a birth cohort $(n=5,979,605)$. Stratification by sex and birthweight percentile (SGA, $<5$ th or 5-10th percentile; appropriate size for gestational age [GA], >10th to $<90$ th percentile; LGA, either $90-95$ th or $>95$ th 
percentile) preceded Cochran-Mantel-Haenszel analysis for GA effect, and multivariate analysis.

Results Autism risk was increased in preterm SGA ( $<5$ th percentile) infants 23-31 weeks (adjusted odds ratio [aOR], 1.60; 95\% confidence interval [Cl], 1.092.35) and 32-33 weeks (aOR, 1.83; 95\% Cl, 1.162.87), and term LGA (>95th percentile) infants 39-41 weeks (aOR, 1.16; 95\% Cl, 1.08-1.26), but was decreased in preterm LGA infants 23-31 weeks (aOR, $0.45 ; 95 \% \mathrm{Cl}, 0.21-0.95)$.

Conclusion SGA was associated with autism in preterm infants, while LGA demonstrated dichotomous risk by GA, with increased risk at term, and decreased risk in the premature infants. These findings likely reflect disparate pathophysiologies, and should influence prenatal counseling, pediatric autism screening, and further autism research.

\section{Repeat antenatal glucocorticoids for women at risk of preterm birth: a Cochrane Systematic Review}

Presented at the 15th Annual Congress of the Perinatal Society of Australia and New Zealand, Hobart, Tasmania, Australia, April 10-13, 2011.

Christopher J.D. McKinlay, MBChBa , Caroline A. Crowther, MD卢, Philippa Middleton, MPH므, Jane E. Harding, DPhila

Administration of antenatal glucocorticoids to women at risk of preterm birth has major benefits for infants but the use of repeat dose(s) is controversial. We performed a systematic review of randomized trials, using standard Cochrane methodology, to assess the effectiveness and safety of 1 or more repeat doses given to women at risk of preterm birth 7 or more days after an initial course. Ten trials were included involving over 4730 women and 5700 infants. Treatment with repeat dose(s) compared with no repeat treatment reduced the risk of respiratory distress syndrome (risk ratio, 0.83; 95\% confidence interval, 0.75-0.91) and serious neonatal morbidity (risk ratio, $0.84 ; 95 \%$ confidence interval, 0.75-0.94). At 2- to 3-year followup (4 trials, 4170 children), there was no evidence of either significant benefit or harm. Repeat doses of glucocorticoids should be considered in women at risk of preterm birth 7 or more days after an initial course, in view of the neonatal benefits.
Inflammation promotes a cytokine response and disrupts the cervical epithelial barrier: a possible mechanism of premature cervical remodeling and preterm birth

Presented orally at the 32nd annual meeting of the Society for Maternal-Fetal Medicine, Dallas, TX, Feb. 6-11, 2012.

Christopher Nold, MD, Lauren Anton, PhD, Amy Brown, PhD, Michal Elovitz, MD

Maternal and Child Health Research Program, Department of Obstetrics and Gynecology, Center for Research on Reproduction and Women's Health, University of Pennsylvania School of Medicine, Philadelphia, PA

Received 28 November 2011. Revised 20 December 2011. Accepted 27 December 2011. Available online 12 February 2012.

Objective An inflammatory challenge disrupts the cervical epithelial barrier and promotes cervical remodeling.

Study Design Immortalized ectocervical and endocervical cells were treated with lipopolysaccharide (LPS), and interleukin (IL)-6, IL-8, and soluble Ecadherin (SECAD) were assessed. Cells were then pretreated with dexamethasone prior to LPS exposure, and IL-6, IL-8, and SECAD levels were again assessed. The integrity of the epithelial cell barrier was determined using a permeability assay.

Results LPS significantly increased IL-6 and IL-8 levels, and SECAD was significantly increased at 24 hours. LPS induced inflammation increased permeability for both cell lines. Dexamethasone pretreatment prior to LPS exposure significantly decreased IL-6 and IL-8 levels in both cell lines. There was no reduction in SECAD levels with dexamethasone pretreatment. Permeability decreased in the presence of dexamethasone for ectocervical cells only.

Conclusion These studies demonstrate an inflammatory challenge to cervical epithelial cells promotes a cytokine release and functionally alters the cervical epithelial barrier. 
Is comprehensive surgical staging needed for thorough evaluation of early-stage ovarian carcinoma?

Presented as a poster at the Annual Meeting on Woman's Cancer of the Society of Gynecologic Oncology, San Francisco, CA, March 14-16, 2009.

Arlene E. Garcia-Soto, MD, Todd Boren, MD, Shana N. Wingo, MD, Thomas Heffernen, MD, David S. Miller, MD

Division of Gynecologic Oncology, Department of Obstetrics and Gynecology, University of Texas Southwestern Medical Center, Dallas, TX

Received 29 March 2011. Revised 11 May 2011. Accepted 16 August 2011. Available online 22 August 2011.

Objective Patients with ovarian cancer may have occult metastasis at the time of surgery. Our purpose was to determine the prevalence and sites of occult metastasis in epithelial ovarian cancer grossly confined to the ovary and examine the significance of routine omentectomy and peritoneal biopsies as part of a comprehensive staging procedure.

Study Design Data were retrospectively abstracted from patients presenting to University of Texas Southwestern Medical Center Hospitals from 1993 through 2009 with ovarian cancer without gross spread beyond the ovary who underwent comprehensive surgical staging.

Results A total of 86 patients with ovarian cancer grossly confined to the ovary who underwent complete surgical staging were identified. Of patients, $29 \%$ were upstaged following comprehensive surgical staging; $6 \%$ had metastatic disease in uterus and/or fallopian tubes, $6 \%$ in lymph nodes, and $17 \%$ in peritoneal, omental, or adhesion biopsies.

Conclusion Patients with epithelial ovarian cancer should continue to undergo comprehensive surgical staging, since it identifies occult metastasis in a significant number of patients.

Vaginal progesterone in women with an asymptomatic sonographic short cervix in the midtrimester decreases preterm delivery and neonatal morbidity: a systematic review and metaanalysis of individual patient data

Roberto Romero, MDaa, Kypros Nicolaides, MD므, Agustin Conde-Agudelo, MD, MPHā, Ann Tabor, MD토

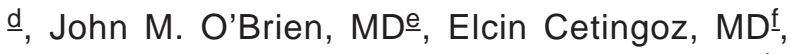

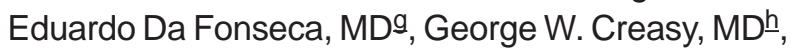
Katharina Klein, MDí, Line Rode, MD뜨, Priya Soma-

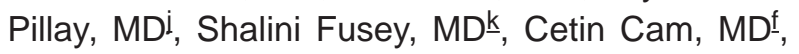
Zarko Alfirevic, MD!, Sonia S. Hassan, MD므, a

Objective To determine whether the use of vaginal progesterone in asymptomatic women with a sonographic short cervix (d"25 mm) in the midtrimester reduces the risk of preterm birth and improves neonatal morbidity and mortality.

Study Design Individual patient data metaanalysis of randomized controlled trials.

Results Five trials of high quality were included with a total of 775 women and 827 infants. Treatment with vaginal progesterone was associated with a significant reduction in the rate of preterm birth $<33$ weeks (relative risk [RR], 0.58; 95\% confidence interval [Cl], $0.42-0.80$ ), $<35$ weeks (RR, $0.69 ; 95 \% \mathrm{Cl}, 0.55-0.88$ ), and $<28$ weeks (RR, 0.50; 95\% Cl, 0.30-0.81); respiratory distress syndrome $(\mathrm{RR}, 0.48 ; 95 \% \mathrm{Cl}$, $0.30-0.76)$; composite neonatal morbidity and mortality (RR, $0.57 ; 95 \% \mathrm{Cl}, 0.40-0.81$ ); birthweight $<1500 \mathrm{~g}$ (RR, 0.55; 95\% Cl, 0.38-0.80); admission to neonatal intensive care unit (RR, $0.75 ; 95 \% \mathrm{Cl}, 0.59-$ $0.94)$; and requirement for mechanical ventilation (RR, $0.66 ; 95 \% \mathrm{Cl}, 0.44-0.98)$. There were no significant differences between the vaginal progesterone and placebo groups in the rate of adverse maternal events or congenital anomalies.

Conclusion Vaginal progesterone administration to asymptomatic women with a sonographic short cervix reduces the risk of preterm birth and neonatal morbidity and mortality.

Early menstrual characteristics associated with subsequent diagnosis of endometriosis

Susan A. Treloar, PhD $\underline{a}, \underline{b}$, Tanya A. Bell, PhD $\underline{c}$, Christina M. Nagle, PhD $\underline{b}$, David M. Purdie, PhD $\underline{d}$, Adèle C. Green, MBBS, PhD $\underline{b}$

Objective The aim of this study was to investigate the association between early menstrual characteristics, before symptom onset, and later diagnosis of endometriosis.

Study Design This was a case-control study of 268 Australian women with surgically confirmed moderateto-severe endometriosis (cases) and 244 women 
without endometriosis (controls). Early menstrual cycle characteristics, before age at symptom onset, were analyzed.

Results Menarche after age 14 years was strongly and inversely associated with endometriosis (odds ratio, $0.3 ; 95 \%$ confidence interval, $0.1-0.6$ ). A history of dysmenorrhea was associated with subsequent endometriosis (odds ratio, 2.6; 95\% confidence interval, 1.1-6.2). Despite a suggestive trend, shorter menstrual cycle length was not associated with endometriosis. Duration of natural menstruation and heaviness of flow were not associated with subsequent risk of endometriosis; neither was the reported type of sanitary protection used nor history of sexual intercourse during menstruation.

Conclusion There is a decreased risk of endometriosis with late age at menarche and an increased risk in women who report an early history of dysmenorrhea. 